Appl. Phys. B 50, 409-413 (1990)

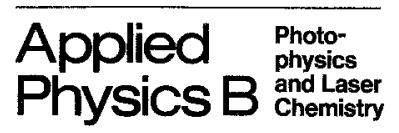

(C) Springer-Verlag 1990

\title{
Theory of Active-Passive Mode-Locking of Solid-State Lasers Using a Nonlinear Mirror
}

\author{
V. P. Petrov ${ }^{1}$ and K. A. Stankov ${ }^{2 \star}$ \\ ${ }^{1}$ Department of Quantum Electronics, Faculty of Physics, University of Sofia, \\ BG-1126 Sofia, Bulgaria \\ ${ }^{2}$ Max-Planck-Institut für biophysikalische Chemie, Abteilung Laserphysik, Am \\ Fassberg, D-3400 Göttingen, Fed. Rep. Germany
}

Received 8 September 1989/Accepted 10 November 1989

\begin{abstract}
The theory of active-passive mode-locked solid-state lasers is developed where the passive mode-locking is achieved by a nonlinear mirror. Steady-state cw numerical solutions are analyzed in detail for the case of a Nd:YAG laser. The steady-state pulse duration can be reduced by an order of magnitude as compared to the pure active modelocking regime.
\end{abstract}

PACS: $42.65,42.55$

Recently a new technique of mode-locking was proposed [1] and experimentally verified on a $\mathrm{Nd}: \mathrm{YAG}$ laser $[2,3]$. The new mode-locking device consists of a nonlinear crystal and a dichroic mirror which replaces the output coupler and is usually highly reflective at the second harmonic. Such a combination increases its reflectivity at the fundamental when increasing the intensity. This so-called nonlinear mirror is characterised by the reflectivity of the dichroic mirror at the fundamental and by the efficiency of the conversion into the second harmonic in the crystal used.

The pulse-shortening action of the nonlinear mirror in a single transit [4] showed comparable behaviour to that of a fast saturable absorber. In comparison with saturable absorbers the new device is remarkable not only for its durability and large spectral range of application but also for its very fast time response.

In a mode-locked $\mathrm{Nd}$ : YAG laser [3] pulse lengths as short as $40 \mathrm{ps}$ at the second harmonic were measured in the second half of the pulse train consisting of approximately 30 pulses. In this laser no etalon was present and the lower limit on the pulse duration is set probably by the limited number of round trips. That is why it becomes very important to analyse the whole

* On leave from Department of Quantum Electronics, Faculty of Physics, University of Sofia, BG-1126 Sofia, Bulgaria mode-locking process over longer evolution periods and to determine the limits of this type of modelocking.

The aim of the present paper is to develop a model for the action of the nonlinear mirror in the $\mathrm{cw}$ or pulsed regime. We study in detail only the $\mathrm{cw}$ regime but we believe the analysis gives an insight into the processes involved also in the pulsed regime in the case of long pulse trains. Since preliminary experiments on pure passive $\mathrm{cw}$ mode-locking were unsuccessful, we have included an acousto-optical modulator in the model. It is known that such combined systems are characterised by improved reliability and stability in the case of saturable absorbers [5].

The present study is restricted to resonant solutions; chirping effects are not considered although they can be adequately described by the model. Both homogeneously and inhomogeneously broadened lasers can be studied by the model. Our main analysis concentrates on the Nd:YAG laser. In addition we give some estimations for the applicability of the new mode-locking technique to presently available $\mathrm{cw}$ actively mode-locked systems.

\section{Theory}

We base our theory on the cavity model shown in Fig. 1. In order to determine the shortest pulses 


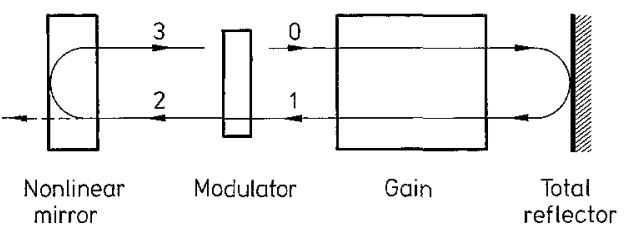

Fig. 1. The model laser cavity. The numbers denote the position of the pulse which travels one round trip in the clockwise direction. The condition for cw steady-state reproduction can, in principle, be imposed at each of these positions

achievable in such systems we describe the active medium by the density matrix equations for a two-level system, which is a good approximation during the interaction with an ultrashort light pulse:

$\left(\partial / \partial t-i \omega_{21}\right) \varrho_{12}=(\mathrm{i} \mu / \hbar) \Delta E-\varrho_{12} / \tau$

$\partial \Delta / \partial t=(2 \mathrm{i} \mu / \hbar)\left(\varrho_{12}-\varrho_{21}\right) E$.

In the above two equations $\Delta=\varrho_{22}-\varrho_{11}$ and $\varrho_{21}=\left(\varrho_{12}\right)^{*} ; \mu$ denotes the transition moment, $\omega_{21}$ the transition centre frequency, and $\tau$ the transversal (phase) relaxation time. The longitudinal relaxation can be neglected since it is much longer than the pulse duration. The slowly varying components of the electric field and the off-diagonal density matrix element can be introduced in the following form:

$E(z, t)=1 / 2\{A(z, t) \exp [\mathrm{i} \phi(z, t)]+c . c$.

$\varrho_{12}(z, t)=\frac{d(z, t)}{2} \exp \{\mathrm{i}[\phi(z, t)+\psi(z, t)]\}$,

where $\phi(z, t)=\omega_{\mathrm{L}} t-k_{\mathrm{L}} z+\varphi(z, t)$ is the phase. The notation can be considerably simplified if the normalized quantities $\bar{E}=(\mu \tau A / \hbar) \mathrm{e}^{\mathrm{i} \varphi}$ and $\bar{\varrho}=d \mathrm{e}^{\mathrm{i}(\varphi+\varphi)}$ together with the complex lineshape function $\mathscr{L}_{21}=\left[1+\mathrm{i} \tau\left(\omega_{\mathrm{L}}-\omega_{21}\right)\right]^{-1}$ and the local coordinates $\zeta=z$ and $\eta=(t-z / v) / \tau$ are used. In these variables the reduced (first order) wave equation assuming linear polarization and propagation in the $z$-direction reads:

$\partial \bar{E} / \partial \zeta=g \bar{Q}$.

In the above equation $g=k_{\mathrm{L}} N_{0} \mu^{2} \tau / 2 \varepsilon_{0} \hbar$, with dimensions of inverse length, is an amplitude amplification coefficient, $N_{0}$ is the number density of inverted ions. The velocity $v$ is assumed to be equal to the group velocity determined by the host and is not influenced by the active ions.

A very important assumption which is justified in particular for solid state lasers is that saturation of the gain cannot occur as a result of a single interaction with the pulse. Thus we can neglect (2) and set $\Delta=1$ in (1). Equation (1) can be now solved for $\bar{\varrho}$ and substituted in (5):

$\partial \bar{E}(\zeta, \eta) / \partial \zeta=g \int_{-\infty}^{\eta} d \eta / \bar{E}\left(\zeta, \eta^{\prime}\right) \exp \left[-\left(\eta-\eta^{\prime}\right) / \mathscr{L}_{21}\right]$.
The solution to the above equation can be expressed as:

$\bar{E}_{1}(\eta)=\bar{E}_{0}(\eta)+2 g \zeta J(\zeta, \eta)$

with

$$
\begin{aligned}
J(\zeta, \eta)= & \int_{-\infty}^{\eta} d \eta^{\prime} \\
& \times \frac{\bar{E}_{0}\left(\eta^{\prime}\right) I_{1}\left[2 \sqrt{g \zeta\left(\eta-\eta^{\prime}\right)}\right] \exp \left[-\left(\eta-\eta^{\prime}\right) / \mathscr{L}_{21}\right]}{2 \sqrt{g \zeta\left(\eta-\eta^{\prime}\right)}},
\end{aligned}
$$

where $I_{1}$ is the modified Bessel function. The subscripts " 0 " and " 1 " of $\bar{E}$ denote the position of the pulse according to Fig. 1 if $\zeta$ is the length of the active medium. Equation (7) is a generalization of the result obtained in [6] for the off-resonant case. We note that since saturation has been neglected (7) describes not only homogeneous but also inhomogeneous broadening provided the inhomogeneous lineshape is of Lorentzian type. The latter is a rather insignificant assumption and obviously inhomogeneously broadened systems can be treated by redefining $\tau$ as the inverse of the total spectral linewidth.

The interaction with the acousto-optical modulator is described in the usual manner [7]

$\bar{E}_{2}(\eta)=\bar{E}_{1}(\eta) \exp \left\{-\delta \sin ^{2}\left[\Omega\left(\eta-\eta_{0}\right)\right]\right\}$,

where the normalized frequency is $\Omega=\tau \omega_{\mathrm{m}}$ ( $\omega_{\mathrm{m}}$ is the driving rf applied to the modulator) and $\delta$ is the depth of modulation.

The interaction with the nonlinear mirror can be accounted for by an intensity-dependent amplitude reflection coefficient [1]:

$\bar{E}_{\mathrm{a}}(\eta)=R_{\mathrm{NL}}(\eta) \bar{E}_{2}(\eta)$,

where $R_{\mathrm{NL}}^{2}=\beta\left(1-\tanh ^{2} A\right), \quad A=\sqrt{\beta} \operatorname{arctanh} \quad(\sqrt{\kappa})$ $-\operatorname{arctanh}(\sqrt{\kappa / \beta})$ and $\beta=\kappa+(1-\kappa) R_{0}^{2} . R_{0}$ is the corresponding value of $R_{\mathrm{NL}}$ at zero intensity and $\kappa$ is the SHG efficiency determined by $\kappa=\tanh ^{2}$ $\left[\sqrt{m|E(\eta)|^{2}} \operatorname{arctanh}\left(\sqrt{\kappa_{0}}\right)\right]$ where $\kappa_{0}$ is related to the intensity used for normalization of the variables and $m$ is a telescoping factor accounting for a possible focusing in the SHG crystal. We note that according to our definition of $\bar{E}, \kappa_{0}$ corresponds to the intensity $I_{0}=\hbar \omega_{\mathrm{L}} / \sigma \tau$ where $\sigma=\mu_{0} \omega_{\mathrm{L}}^{2} \mu^{2} \tau / \hbar k_{\mathrm{L}}$ is the maximum interaction cross section and the normalized (by $I_{0}$ ) intensity used further is simply $I=|\bar{E}|^{2}$.

Equations (7), (8), and (9) if iterated can be used to model a pulsed or a cw system. The aim of the present paper is, however, to study the $\mathrm{cw}$ active-passive regime only and that is why we make some additional simplifications. In the $\mathrm{cw}$ regime each resonator element modifies the field envelope only slightly, exactly as in the case of passively mode-locked dye lasers [8]. 
This approximation means that the position of each element relative to the other elements is unimportant. In cw systems the total losses and consequently the saturated gain $g \zeta$ are small compared to unity. The bandwidths of the transitions are, at least for solidstate lasers, much larger than the modulation frequency and the expression for the modulator $(B)$ can also be expanded since $\Omega$ is a very small number. Following this concept for the nonlinear mirror $\left(R_{0}=1-\gamma\right)$ we arrive at the self-consistency equation

$$
\begin{aligned}
\bar{E}(\eta)= & \left\{1+2 g \zeta J(\zeta, \eta) / \bar{E}(\eta)-\delta \Omega^{2}\left(\eta-\eta_{0}\right)^{2}\right. \\
& -\gamma(1-\kappa)\} \bar{E}(\eta) \mathrm{e}^{\mathrm{i} \chi} .
\end{aligned}
$$

We have not included any time shift in the complex amplitude after one full round trip [left side of (10)]. This small time shift, in analogy with the hybridly mode-locked dye lasers [9], should vanish since the active modulator fixes a time origin at $\eta_{0}$. However, we denote by $h$ the time delay caused by the active medium alone, which should be compensated. The additional phase shift $\chi$ is insignificant and accounts for a possible deviation of the propagation constant from the assumed linear value $k_{\mathrm{L}}=\omega_{\mathrm{L}} n / \mathrm{c}$.

The model can be completed now if a certain saturation scheme is assumed in order to limit the energy. The situation is different for homogeneous and inhomogeneous broadening [7] and in general spectral decomposition of the field is needed. We assume a rather simple expression for $g$ following [10]:

$g=g_{0} /\left(1+T_{21} \mathscr{E} / U\right)$

In (11) $g_{0}$ is the small signal gain, $T_{21}$ is the upper state lifetime, $U$ the round trip time, and $\mathscr{E}=\int_{-\infty}^{\infty} I(\eta) d \eta$ is the normalized intracavity energy. The exact consideration of the saturation mechanism would only slightly modify the value of $g_{0} \zeta$ for which the calculated results are correct. That is why we believe (11) is a good approximation for both homogeneously and inhomogeneously broadened lasers.

Steady-state solutions can be generated now by iterating equation (10) with (11) after a certain number of round trips. We do not consider here transient effects and the steady-state pulse is independent of the input signal injected. It is characterised by high stability and deviation of $<0.01 \%$ for integral parameters like energy, duration, asymmetry etc. from round trip to round trip.

\section{Results and Discussion}

We specify first of all the parameters used in this section since they are representative for the most commonly used Nd:YAG laser. Using the data for the laser bandwidth $(120 \mathrm{GHz})$, the maximum cross section $\left(8.810^{-19} \mathrm{~cm}^{2}\right)$ and the upper state lifetime ( $230 \mu \mathrm{s}$ ) we get $\tau=2.65 \mathrm{ps}$ (which is our scaling parameter in the dimensionless pulse duration $T$ measured at FWHM intensity), $\Omega=0.00083$ and $T_{21} / U=23000$ if a round trip time $U=10 \mathrm{~ns}$ is assumed. From the above values it follows that the dimensionless intensity $I$ is normalized to the value $3.8210^{4} \mathrm{MW} / \mathrm{cm}^{2}$ and the dimensionless energy density $\mathscr{E}$ to the value $100 \mathrm{~mJ} / \mathrm{cm}^{2}$. The modulation depth is $\delta=2$ and for the nonlinear mirror we assume $\gamma=0.1$ and a focusing factor $m=10$. We suppose that the laser rod and the SHG crystal are antireflection coated and neglect any other linear losses in order to achieve maximum intracavity intensity. The inclusion of additional linear losses in the model is not expected to substantially modify the results if they are balanced by higher gain. In order to estimate $\kappa_{0}$ we used the experimentally achievable values for KTP [11] which amount to about $25 \%$ of the theoretical limit. For a crystal length of $0.6 \mathrm{~cm}$ we get $K=\operatorname{arctanh}\left(\sqrt{\kappa_{0}}\right)=11.7$. Finally we assume the carrier frequency of the pulse to be centered at the line maximum, i.e. $\omega_{\mathrm{L}}=\omega_{21}$, which leads to real steady-state solutions. The zero point of the active modulator can be chosen at $\eta_{0}=0$. We note, however, that, for the chosen set of parameters, the modulator has a very weak influence as a pulse shortening mechanism or as a factor compensating for the time shift caused by the active medium. This is due to the fact that our numerical consideration is limited in the interval $|\eta|<20$. Thus it can be assumed that the acousto-optical modulator plays a role only in the initial stages of the selection of the pulse from the noise and as a stabilizing mechanism for the repetition frequency. The main shortening mechanism in the case considered is the nonlinear mirror and its action is balanced by the broadening action of the active medium. The active medium not only disperses the pulse due to its spectral selection but is the reason for the asymmetrical steady-state profiles and a time delay. The asymmetry follows from the presence of an imaginary part in the Fourier image of (6) even at $\mathscr{L}_{21}=1$. We note that even in the limit of pure active mode-locking the solution should be asymmetric and the Gaussian solution of [7] is a result of the parabolic expansion of the gain function. The symmetric solutions obtained in [6] on the other hand are due to the fact that the pulse length is comparable to the cavity round trip time for the set of parameters chosen there.

The pulse delay caused in our case by the active medium can be compensated only by cavity detuning. The length by which the cavity should be shortened can be calculated to be equal to $h \tau c$ and since we shift the pulse in order to compensate for the delay our 

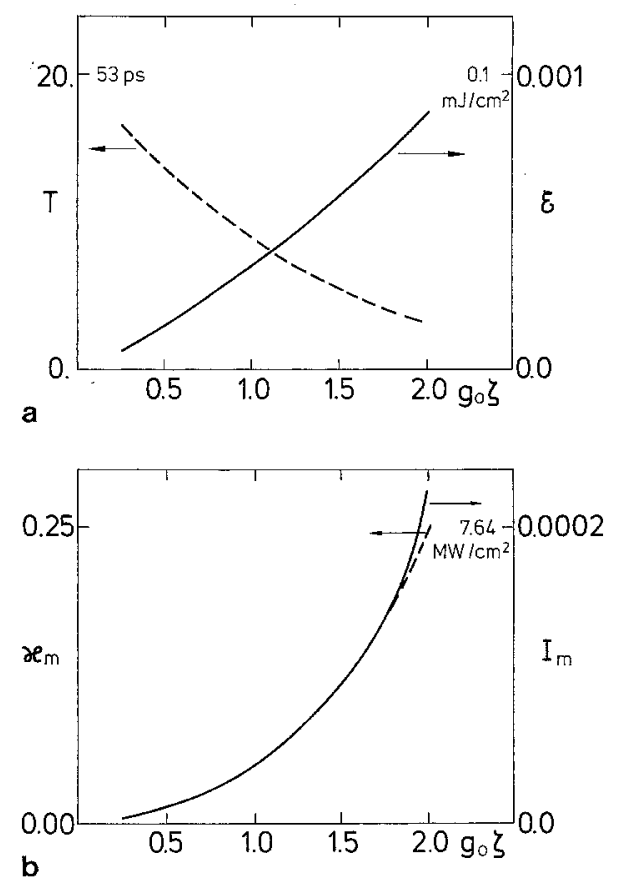
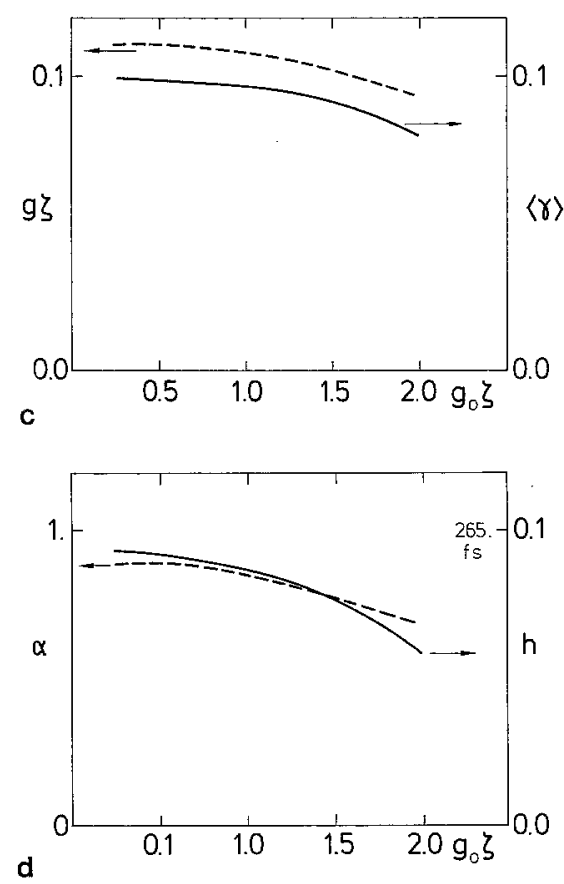

Fig. 2a-d. Steady-state pulse parameters versus small signal gain. For the normalized quantities a second real physical scale is given in addition which clarifies the results for the Nd:YAG example. (a) Intracavity pulse energy density $\mathscr{E}$ (solid line) and pulse duration $T$ (dashed line). (b) Maximum intensity $I_{\mathrm{m}}$ (solid line) and conversion efficiency $\kappa_{\mathrm{m}}$ (dashed line) calculated at $I_{\mathrm{m}}$. (c) Mean loss $\langle\gamma\rangle$ (solid line) and steady-state saturated gain $g \zeta$ (dashed line). (d) Pulse delay in the active medium $h$ (solid line) and pulse asymmetry $\alpha$ (dashed line) numerical method gives results also for the delay $h$ experienced in the passage through the active medium.

All steady-state pulse parameters are plotted in Fig. 2. $\kappa_{\mathrm{m}}$ is the SHG efficiency calculated at the resulting maximum intensity of the pulse. $\alpha$ is the asymmetry calculated as a ratio of the widths of the leading and trailing edges of the pulse measured at half maximum intensity. Since the mirror losses are nonlinear we define a mean amplitude loss coefficient $\langle\gamma\rangle=\mathscr{E}_{\mathrm{L}} / 2 \mathscr{E}$ where $\mathscr{E}_{\mathrm{L}}$ is the energy lost in each reflection.

As can be seen from Fig. 2a the increase of the small signal gain $g_{0} \zeta$ results in constant increase of the intracavity pulse energy $\mathscr{E}$ and reduction of the pulse duration $T$ due to the enhanced mode-locking action of the nonlinear mirror. The limit of pure active modelocking ( $\left.g_{0} \zeta \Rightarrow 0\right)$ could not be reached in the time interval considered but according to the analytical results in [7] it corresponds to $T=27.36(72.5 \mathrm{ps})$. We see that even very low conversion efficiencies $\left(\kappa_{\mathrm{m}}=0.014\right.$ at $\left.g_{0} \zeta=0.5\right)$ reduce the pulse duration nearly twice: $T=13.6$ (36 ps). Since we have chosen exactly the same laser parameters it is possible to compare our results with those obtained by an approximate analytical treatment in [12]. For the case considered in the first row of [Ref. 12; Table 1] $\kappa_{\mathrm{m}}=0.1$ and we obtain such a value of $\kappa_{\mathrm{m}}$ at $\mathrm{g}_{0} \zeta=1.39$ where $T=6.2(16.43 \mathrm{ps})$. This value of $T$ is about $20 \%$ shorter than the corresponding value in [12]. We note that the differences arise from the approximate expressions used in [12] to describe the active medium and the nonlinear mirror.
The pulse leading edge is always steeper and the asymmetry tends to 1 when $g_{0} \zeta \Rightarrow 0$. The established saturated gain $g \zeta$ is greater than the mean loss $\langle\gamma\rangle$ but tends to follow the dependence of $\langle\gamma\rangle$. The pulse is always delayed by the active medium but this delay is smaller at shorter pulse durations. A comparison of the curves for $I_{\mathrm{m}}$ and $\kappa_{\mathrm{m}}$ shows that $\kappa_{\mathrm{m}}$ closely follows $I_{\mathrm{m}}$ and saturation occurs only at high intensities $\left(g_{0} \zeta>1.8\right)$. This can be easily explained on the basis of our assumption made for $R_{\mathrm{NL}}$ in (10). We used $\gamma$ as a small parameter and assumed $R_{\mathrm{NL}}=\sqrt{\beta}=1-\gamma(1-\kappa)$ since $\tanh ^{2} A \propto A^{2} \propto \gamma^{2}$ (see the bottom curve on [Ref. 1; Fig. 3] or on [Ref. 12; Fig. 1a]. Thus we preserved the nonlinear dependence of $\kappa$ on $I$ but for small intensities $\kappa$ can also be expanded and we obtain $\kappa=K^{2} m I-2 K^{4} m^{2} I^{2} / 3$. In such a way at high values of $I_{\mathrm{m}}$ the quadratic term gives a correction which is responsible for the saturation of $\kappa_{\mathrm{m}}$. We note that if $\kappa$ is linear in $I$ (only the first term preserved) the description of the nonlinear mirror for small $\gamma$ is equivalent to the description of the saturable absorber developed in the theory of passive mode-locking by absorbers [10]. This theory gives a sech ${ }^{2}$ solution for the steady-state pulse intensity. The symmetric analytical solution is, however, achieved in [10] again through parabolic fit of the gain profile and that is why it cannot be compared to our solution even for low values of $I_{\mathrm{m}}$.

In Fig. 3 we have plotted the steady-state pulse profiles obtained for several values of the small signal gain $g_{0} \zeta$.

Finally we want to make some estimates for commercially available and/or very powerful solid- 


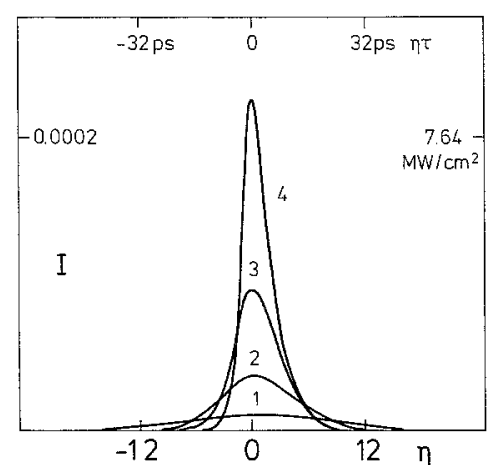

Fig. 3. Steady-state intensity profiles for sveral values of the small signal gain: $g_{0} \zeta=0.5$ (curve 1), $g_{0} \zeta=1$. (curve 2), $g_{0} \zeta=1.5$ (curve 3), and $g_{0} \zeta=2$ (curve 4)

state systems. The most powerful commercially available actively mode-locked Nd:YAG laser, the Antares [13], delivers about $22 \mathrm{~W}$ of mean mode-locked power at $100 \mathrm{ps}$ pulse duration. The peak intracavity intensity can be estimated to be of the order of $0.7 \mathrm{MW} / \mathrm{cm}^{2}$. For the threshold of the passive mode-locking effects $\left(g_{0} \zeta=0.5\right.$ or a pulse shortening by a factor of 2$)$ we have from Fig. $2 m I_{\mathrm{m}}=4 \mathrm{MW} / \mathrm{cm}^{2}$. Since this last figure corresponds to a duration that is already halved we can conclude that a telescoping by $m \geqq 3$ would be sufficient for this laser to enter the combined modelocking regime and halve its pulse width.

Even higher unfocused intracavity powers can be achieved in systems based on other host materials which exhibit larger available bandwidth. The laser based on Nd:YLF [14] exhibits peak unfocused intracavity intensity $>1.3 \mathrm{MW} / \mathrm{cm}^{2}$ at pulse duration $35 \mathrm{ps}$. Such a system seems very promising when modelocked at higher frequencies and pumped by laser diodes. The authors of [15] achieved durations of $<9 \mathrm{ps}$ by acousto-optical mode-locking of Nd:YLF at $500 \mathrm{MHz}$. Although the mean power is lower $(100 \mathrm{~mW}$ out of the cavity), tight focusing in the crystal is possible in such schemes.

Very high peak intensities are also available in $\mathrm{cw}$ mode-locked $\mathrm{Nd}$ :phosphate glass lasers [16]. The focused intensity in the active medium pumped by a cw $\mathrm{Ar}^{+}$laser amounts [16] to $30 \mathrm{MW} / \mathrm{cm}^{2}$ for the $10 \mathrm{ps}$ pulse length achieved. This means that if focusing of the same order of magnitude is applied to the SHG crystal, this would be sufficient for passive modelocking of this system, too.

\section{Conclusion}

We have presented an analysis of active-passive modelocking of solid-state lasers with the addition of a nonlinear mirror acting as a passive mode-locking device. At high pump rates the steady-state pulses can be considerably shorter (more than one order of magnitude) than the corresponding durations for pure active mode-locking. The pulse duration limit is set in the present model by the available bandwidth and should be of the order of the phase relaxation time $\tau$. To investigate extreme pulse shortening it is necessary to include dispersion and self-phase modulation effects, i.e. chirping of the steady-state pulse $[8,9]$. These effects are always present at higher intensities. Group velocity mismatch in the SHG crystal should also be taken into account for very short pulses. We intend to study all these problems as well as transient behaviour of such a hybrid system in a forthcoming publication.

Acknowledgement. The authors are indebted to V. D. Stoev who performed much of the numerical work for the present papers. K. Stankov acknowledges a research fellowship from the Alexander von Humboldt Foundation.

\section{References}

1. K.A. Stankov: Appl. Phys. B 45, 191 (1988)

2. K.A. Stankov, J. Jethwa: Opt. Commun. 66, 41 (1988)

3. K.A. Stankov: Opt. Lett. 14, 359 (1989)

4. K.A. Stankov: Appl. Opt. 28, 342 (1989)

5. H.P. Kortz: IEEE J. QE-19, 578 (1983)

6. P.M. Radmore: Phys. Lett. 89 A, 4 (1982)

7. D.J. Kuizenga, A.E. Siegman: IEEE J. QE-6, 694 (1970)

8. V. Petrov, W. Rudolph, B. Wilhelmi: Rev. Phys. Appl. 22, 1639 (1987)

9. V. Petrov, W. Rudolph, U. Stamm, B. Wilhelmi: Phys. Rev. A 40, in press (1989)

10. H.A. Haus: J. Appl. Phys. 46, 3049 (1975)

11. T.A. Driscoll, H.J. Hoffmann, R.E. Stone, P.E. Perkins: J. Opt. Soc. Am. B 3, 683 (1986)

12. J.R.M. Barr: Opt. Commun. 70, 229 (1989)

13. Coherent Laser Products Division, Antares Laser

14. H. Vanherzeele: Appl. Opt. 27, 3608 (1988)

15. U. Keller, K.J. Weingarten, K.D. Li, D.C. Gerstenberger, P.T. Khuri-Yakub, D.M. Bloom: CLEO'89, post deadline paper PD2

16. L. Yan, J.-D. Ling, P.-T. Ho, C.H. Lee, G.L. Burdge: IEEE J. QE-24, 418 (1988) 